\title{
La Formación de la Ciudadanía en el Marco de la Agenda 2030 y la Justicia Ambiental
}

\section{Citizenship Education in the Framework of 2030 Agenda and Environmental Justice}

\author{
M. Ángeles Murga-Menoyo * \\ Universidad Nacional de Educación a Distancia, España
}

\begin{abstract}
Este artículo busca analizar las orientaciones de la Unesco para una educación coherente con los Objetivos de Desarrollo Sostenible (ODS) señalados en la agenda; identificar sus debilidades y fortalezas para la formación de una ciudadanía comprometida con la justicia ambiental; y proponer una alternativa para subsanar las deficiencias identificadas. Se ha utilizado una metodología hermenéutica aplicada al estudio de la bibliografía y documentos. Los resultados indican que las directrices formativas de Unesco no agotan las finalidades prioritarias que el movimiento por la justicia ambiental atribuye a la educación, aunque pueden contribuir a ellas; las respectivas fortalezas de ambos enfoques podrían dar lugar a sinergias con efectos recíprocamente beneficiosos. En el artículo se destacan las características significativas de la Agenda 2030 y el concepto de justicia ambiental. Se analizan las competencias clave en sostenibilidad que la Unesco propone y se valoran desde la perspectiva de la justicia ambiental. El cuarto apartado está dedicado al tipo de ciudadanía cuya formación ambos enfoques precisan. Finalmente, se hace una propuesta integradora sobre las necesidades formativas para alcanzar tanto los ODS como la justicia ambiental.
\end{abstract}

Descriptores: Desarrollo sostenible; Justicia social; Valores sociales; Ética; Educación.

This article seeks to analyze the guidelines of Unesco for an education consistent with the Sustainable Development Objectives (SDO) set out in the agenda; identify their weaknesses and strengths for the education of a citizenship committed to environmental justice; and propose an alternative to remedy the deficiencies identified. A hermeneutic methodology has been used for the analysis of bibliography and documents. The results shown that Unesco's training guidelines do not exhaust the priority objectives that the environmental justice movement attributes to education, although they can contribute to them; the respective strengths of both approaches could lead to synergies with reciprocal beneficial effects. The article highlight the significant features of Agenda 2030 and the concept of environmental justice. The key competences in sustainability that Unesco proposes are analyzed next, and they are evaluated from the perspective of environmental justice. The fourth section is devoted to the type of citizenship that is required for the education considered in both approaches. Finally, we made an integrative proposal about the educational needs to achieve ODS and environmental justice.

Keyword: Sustainable development; Social justice; Social values; Ethics; Education.

\begin{abstract}
Proyecto EDU2015-66591-R financiado por el Ministerio de Economía, Industria y Competitividad del Gobierno de España.
\end{abstract}

*Contacto:mmurga@edu.uned.es

ISSN: 2254-3139

www.rinace.net/riejs/

revistas.uam.es/riejs
Recibido: $\quad 15$ de noviembre 2018

$1^{\text {a }}$ Evaluación: 13 de enero 2018

$2^{\text {a }}$ Evaluación: 9 de marzo 2018

Aceptado: $\quad 16$ de abril 2018 


\section{Introducción}

La segunda mitad del siglo Xx es fecunda en enfoques, movimientos y experiencias sociales innovadoras, algunos de los cuales han llegado evolucionados hasta nuestros días. Entre otros, el movimiento por la justicia ambiental, que nace estrechamente vinculado a la justicia social (Dobson, 1998; Wenz, 1988), y el enfoque del desarrollo sostenible, hoy ampliamente aceptado por la comunidad internacional (ONU, 2015).

La mayoría de estos movimientos tiene su correlato en la educación. Hemos asistido a la emergencia de todo un abanico de educaciones adjetivadas, como son, entre las relacionadas con los mencionados enfoques, la educación para la paz y los derechos humanos, la educación ambiental, la educación para el desarrollo sostenible o la educación por la justicia ambiental, por mencionar algunas de las más difundidas. Las diferencias entre las distintas denominaciones se encuentran a veces en sus matices y énfasis preferentes, aunque en ocasiones resultan significativas e incluso afectan al propio corazón del concepto que fundamenta el enfoque.

En el caso de la educación por la justicia ambiental, en su interés hacia la naturaleza coincide con la educación para el desarrollo sostenible; un enfoque este último que, pretendiendo recoger la tradición de la educación ambiental, hoy está siendo avalado y promovido por los más altos organismos internacionales (ONU, 2015; Unesco, 2014). Sin embargo, teniendo en cuenta que no se trata de enfoques monolíticos -especialmente en el caso del desarrollo sostenible- sino abarcadores de toda una horquilla de posiciones con sus peculiaridades y actuaciones contextualizadas, aunque en algunos aspectos significativos ambos tipos de educación pudieran reforzarse mutuamente, en otros la laguna entre las respectivas prioridades podría convertirlos, en la práctica, en recíprocamente excluyentes.

Es un hecho que en estos momentos la educación para el desarrollo sostenible ocupa un lugar preferente en la agenda educativa internacional institucionalizada; una agenda estrechamente unida al logro de los Objetivos de Desarrollo Sostenible (Agenda 2030) que fueron aprobados por la Asamblea General de las Naciones Unidas para el periodo 2016-2030 (ONU, 2015). Pero, ¿cabe afirmar que es ésta una educación para la justicia ambiental? ¿Qué características debería reunir para que lo fuera? ¿Qué capacidades, valores, actitudes, habilidades, hábitos, o, por expresarlo con un único término hoy profusamente utilizado, competencias, demandan prioritariamente ambos enfoques? ¿Existen similitudes significativas entre la educación para el logro de los ODS y la educación por la justicia ambiental?

Nuestra hipótesis de partida es que la educación para el desarrollo sostenible no agota los planteamientos de la educación para la justicia ambiental, y que esta última, nacida en Estados Unidos y con fuerte presencia en América Latina (en su variante "ecología de los pobres”) aunque menos en Europa, tiene pleno sentido en nuestros días incluso en los países considerados desarrollados, cuya calidad de vida, en amplias capas de la población, hoy parece encontrarse en alto riesgo de retroceso. En consecuencia, defendemos que la formación de la ciudadanía para la sostenibilidad habrá de forjarse en la intersección de ambas perspectivas.

En este trabajo nos proponemos someter a análisis esta hipótesis y avanzar algunas respuestas a los interrogantes mencionados. En primer lugar, describiremos el marco de acción pedagógica que viene dibujado por la Agenda 2030 y los Objetivos de Desarrollo 
Sostenible (2016-2030). A continuación, desatacaremos los aspectos a nuestro juicio más significativos que caracterizan el enfoque de la justicia ambiental, concepto estrechamente co-implicado con el de justicia social, así como las prioridades educativas que corresponden a dicho enfoque. En tercer lugar, analizaremos comparativamente las competencias que la ciudadanía ha de dominar para que ambos enfoques puedan afianzarse socialmente; y, seguidamente, el tipo de ciudadanía que se requiere para ello. Finalmente, un último apartado nos permitirá esbozar una respuesta a la hipótesis inicial y ofrecer un planteamiento integrado, que pueda orientar el diseño de propuestas de acción pedagógica aprovechando las respectivas fortalezas en beneficio de una educación comprometida con las urgentes necesidades sociales de nuestros días.

Hemos adoptado una metodología hermenéutica, procediendo al análisis de una muestra intencional integrada por publicaciones de autores clásicos en el ámbito de la justicia ambiental y, en el caso de los ODS, documentos y declaraciones de Naciones Unidas y la Unesco, ineludibles para entender el alcance y significado de la Agenda 2030. La búsqueda bibliográfica se ha realizado mediante la plataforma Linceo+ que facilita el acceso a medio centenar de bases de datos, entre ellas ERIC, Web of Science y Dialnet, así como a los textos. Como categorías de análisis, y posterior comparación de los marcos teóricos, se han adoptado las siguientes: finalidad del enfoque/movimiento; problemáticas objeto de atención; concepto de derechos humanos; jerarquía axiológica; metas y prioridades educativas.

\section{La agenda 2030: Un marco para la acción pedagógica orientada a la sostenibilidad}

La Agenda 2030 establece las prioridades estratégicas de las Naciones Unidas para los próximos tres lustros (2016-2030), asumiendo la óptica de los derechos humanos como base para justificar la pertinencia de los diecisiete Objetivos de Desarrollo Sostenible (ODS) que señala. Así se recoge en la Resolución por la que fue aprobada, cuyo texto reconoce expresamente que con estas metas se pretende hacer realidad los derechos humanos de todas las personas (ONU, 2015).

Los ODS reflejan los grandes retos a los que hoy se enfrenta la Humanidad derivados de las problemáticas ecológicas, económicas y sociales globales. Todas ellas se han visto aumentadas desde que en los años setenta comenzaran a ser denunciadas, también con creciente insistencia, por foros científicos y organismos internacionales (p. ej., IPPC, 2014; Millennium Ecosystem Assessment, 2005; ONU, 1972, 2002, 2012; UNEP, 2011). Hoy, los ODS se plantean como una hoja de ruta en el camino hacia la sostenibilidad y, como señalan Gil Pérez y Vilches (2017), mantienen con los derechos humanos universales (DDHH) una relación "biyectiva: si el respeto de los derechos es (...) condición indispensable para avanzar en la transición a la Sostenibilidad, esta es necesaria, a su vez, para hacer posible la universalización de los DDHH“ (p. 91); y lo argumentan con acierto, destacando la condición de posibilidad que para la primera suponen estos derechos universales, cuyo contenido sigue evolucionando de forma expansiva.

Pero, si en 2015 pudimos celebrar la aprobación y lanzamiento internacional de la Agenda 2030 como un refuerzo para afianzar en el imaginario social la percepción de las implicaciones recíprocas entre sociedad, ecología y economía, y un impulso para avanzar hacia logros imprescindibles en el marco de la justicia social y ambiental, dos años después 
los avances visibles, más allá de la retórica al uso, se revelan insuficientes tanto para atajar la magnitud de la crisis civilizatoria global como para lograr las metas señaladas por la Asamblea General de las Naciones Unidas (Banco Mundial, 2017; Helliwell, Layard y Sachs, 2016).

Sin embargo, es preciso reconocer que los oDs suponen un avance con relación a sus predecesores, los Objetivos de Desarrollo del Milenio (ODM), algunas de cuyas limitaciones Caride Gómez (2009) señaló con acierto. Las actuales metas han sido reformuladas y ampliadas, haciendo explícita la perspectiva de la sostenibilidad. Merecen, pues, una primera valoración positiva. En primer lugar, porque, mientras los ODM se situaban en un marco de cooperación al desarrollo, dejando al margen la imprescindible transformación sociocultural y económica de los países industrializados, la Agenda 2030 de Naciones Unidas, al exigir la sostenibilidad de los objetivos señalados para lograr la meta del desarrollo, ha ampliado el foco de atención a los países más avanzados desde el punto de vista tecnológico. Con su pronunciamiento quedan convocados a cumplir estas metas todos los países que conforman la comunidad internacional.

$\mathrm{Y}$, en segundo lugar, porque el sistema de indicadores que se ha establecido para evaluar los avances contempla, por primera vez, los impactos que cada país provoca sobre los demás; es decir, su huella ecológica (Sachs et al., 2017). En adelante ningún país será reconocido cumplidor de la Agenda 2030 si no minimiza, hasta eliminarlo, su impacto ecológico y social sobre territorios ajenos. Desde esta perspectiva, cabe considerar que la aprobación de los ODS supone un impulso hacia la universalización, en este aspecto, de la justicia ambiental.

Adicionalmente, en el texto aprobado por las Naciones Unidas (ONU, 2015), se hacen visibles las interrelaciones recíprocas entre las distintas metas, especialmente en el caso de la educación (objetivo 4.), con alusión explícita a la educación para el desarrollo sostenible (objetivo 4.7). Difundir estos objetivos para su conocimiento generalizado, sensibilizar a la población sobre su pertinencia y, sobre todo, orientar los procesos formativos en todos los ámbitos y modalidades de la educación, de tal manera que se alcancen los aprendizajes necesarios para el tipo de ciudadanía que requiere el cumplimiento eficaz de la Agenda 2030, es un reto de envergadura que hoy tienen planteado todos los sistemas educativos.

Aceptando esta premisa, es sin embargo necesario reconocer que, al bajar al terreno concreto de la práctica educativa, la agenda concede a cada país, comunidad y centro de formación plena libertad a la hora de diseñar la acción pedagógica. Y, por este motivo, los énfasis se sitúan, más allá de la retórica que señala su recíproca interdependencia, en aquellos objetivos que presentan mayor afinidad con la posición, aun dentro del enfoque del desarrollo sostenible, dominante en cada caso.

\section{Justicia social y ambiental: implicaciones para la educación}

Dos conceptos con una tradición interrelacionada de cuatro décadas son justicia ambiental y justicia social. El primero de ellos se utiliza para designar la equitativa distribución entre la población (intra-estado e inter-estados) tanto de los beneficios como de los impactos negativos que se derivan de la aplicación de las normativas y políticas nacionales e internacionales en materia de medio ambiente. 
La justicia ambiental pivota sobre la equidad y, por tanto, se sitúa de lleno en el ámbito de la justicia social, la cual, a su vez, tras larga evolución, en la actualidad ha visto consolidarse dicho principio como una de sus dimensiones constitutivas. Como defiende Vicente Jiménez (2002),

\begin{abstract}
la justicia social requiere la determinación de lo que corresponde a cada cual, no sólo en virtud de la dinámica del orden social institucionalizado -es decir, las estructuras sociales en conexión con el régimen jurídico, sino que ahora amplía tal dinamismo, a la funcionalidad dinámica inherente al área abierta de los ecosistemas. (p. 158)
\end{abstract}

Sobre la evolución del concepto de justicia ambiental -y el movimiento que ha contribuido a su difusión- existe una amplia bibliografía (Arriaga Legarda y Pardo Buendía, 2011; Espinosa, 2012; Moreno Jiménez, 2007, 2010; entre otros). El concepto (principio) se consolida en los años noventa; si bien su origen se remonta a los setenta, en Estados Unidos, impulsado por el auge de los movimientos de base vinculados al activismo por los derechos civiles, entre ellos los ambientales. Se fragua en el ámbito local, a raíz de confirmarse, gracias a la insistencia del activismo ciudadano, la relación entre las políticas ambientales y el impacto negativo, significativamente diferente, de las problemáticas ambientales sobre la vida cotidiana y la salud de los grupos sociales más vulnerables. Surge, por tanto, radicalmente asociado a la defensa de los derechos humanos, siendo una peculiar característica que marca su trayectoria la amplia militancia, incluso protagonismo, de las mujeres entre sus miembros.

Hoy son pilares básicos del enfoque, los siguientes: el principio precautorio, la responsabilidad ampliada (inter-generacional e inter-específica), la equidad intergeneracional, la interdependencia persona-comunidad-naturaleza y el empoderamiento de la ciudadanía, que es reconocida como sujeto activo para la toma de decisiones en política ambiental, según las reglas de la democracia a partir de información transparente y rigurosa.

El principal foco de atención que asume la justicia ambiental son los conflictos ecológicos distributivos, originados por dos principales tipos de causas: desigual acceso a los bienes y servicios de la naturaleza, y desigual reparto de la contaminación y los residuos producidos por la actividad humana. El movimiento, nacido a partir de casos locales, ha ido ampliando sus miras hasta adquirir un enfoque global, planetario.

De forma análoga y en fechas similares, aunque con las peculiaridades propias de su contexto cultural, de carácter rural, en Latinoamérica nace el movimiento Ecologismo de los pobres, claramente convergente con aquel en sus principios básicos (Martínez Alier, 2004). Ambos apuestan por un antropocentrismo débil, que reconoce la interdependencia y co-evolución de la especie humana junto con las restantes especies del planeta.

El corpus teórico de la justicia ambiental se inspira en diferentes enfoques socio-culturales, notablemente en el movimiento conservacionista y en el ecologismo, derivándose de esta influencia sus dos principales preocupaciones: la gestión ambiental y la gestión del territorio; ambas cuestiones directamente relacionadas con la justicia y la equidad social (económica, de clase y racial). La primera de ellas, refleja el despertar de la conciencia ciudadana ante las problemáticas por contaminación de las aguas, el aire y los suelos, ocasionadas por la industrialización, y sus muy perniciosos efectos sobre la salud de la población.

A raíz de esta sensibilización, la gestión del territorio se revela como un instrumento político significativo para evitar la desigual distribución social -mediada, en parte, por una 
desigual distribución espacial- de los impactos, tanto negativos como positivos de la industrialización, con perjuicio para los más vulnerables (Moreno Jiménez, 2010). Se acuñan conceptos como, por ejemplo, espacio ambiental, huella ecológica o deuda ecológica, que contribuyen a la construcción teórica del enfoque y facilitan argumentos para justificar sus dos principales metas: una equitativa distribución de los impactos y beneficios de las medidas políticas sobre el medio ambiente, y un uso, igualmente equitativo, de los recursos y bienes de la naturaleza.

Desde su condición originaria como iniciativa ciudadana, el movimiento por la justicia ambiental ha pasado a adquirir una dimensión político-administrativa que se ha visto reflejada en la jurisprudencia.

El concepto ha ido adquiriendo un significado científico y jurídico más aquilatado, (...) está llamado a ocupar un puesto destacado (...) en la evaluación de toda suerte de políticas públicas con dimensión espacial, entre ellas las concernientes a la ordenación urbana y territorial, por cuanto la no discriminación de los ciudadanos (...) constituyen pilares de las democracias. (Moreno Jiménez y Cañada Torrecilla, 2007, pp. 302-303).

En coherencia, pues, con las peculiaridades y finalidades del movimiento por la justicia ambiental, se requiere una educación para el activismo social y el compromiso político. Una educación orientada al compromiso activo de la ciudadanía que permita desactivar a los victimarios personales y estructurales, a nivel local y global. Una educación que, como acertadamente señala Díaz-Salazar (2016),

ha de incidir en todas las áreas existenciales del ser humano: la mente (sentido de la vida), los ojos (visión de la realidad), el corazón (sentimientos y emociones), el estómago o "segundo cerebro" (dolor, indignación, rabia, rebelión), las manos (la acción) y los pies (la movilización). (p. 181)

\section{La formación de competencias para la sostenibilidad}

Pero, ¿puede un mismo modelo educativo atender los requerimientos del desarrollo sostenible y la justicia ambiental? ¿Existe complementariedad entre las metas y prioridades educativas de ambos enfoques? ¿Podría el tipo de educación que Unesco promueve para el logro de la Agenda 2030 y los ODS dar respuesta a la formación de la ciudadanía que exigen ambos enfoques? Son las cuestiones que a continuación abordaremos.

Unesco señala que en el marco contextual de los oDs el papel de la educación, recogiendo y ampliando la larga tradición de la educación ambiental como posibilitadora de la sostenibilidad, es contribuir a cada uno de ellos con objetivos de aprendizaje específicos en tres principales dominios: cognitivo, socioemocional y conductual (Unesco, 2017). En el dominio cognitivo, facilitando el conocimiento y las habilidades de pensamiento necesarias para una mejor comprensión de los ODS y los retos que suponen. En el dominio socioemocional, consolidando las habilidades sociales que permiten a los estudiantes colaborar, negociar y comunicarse para promover los ODS, así como las habilidades de auto-reflexión, valores, actitudes y motivación facilitadoras del aprendizaje autónomo. Finalmente, en el dominio conductual, se atribuye a la educación la misión de formar competencias para la acción orientada hacia la sostenibilidad.

En ese mismo texto, referencia obligada por su actualidad y autoría, la Unesco propone que se integren nuevos contenidos en el currículo -como son: el cambio climático, la 
pobreza o el consumo sostenible- y que se primen los entornos interactivos centrados en el estudiante y en el proceso de enseñanza-aprendizaje. Defiende, así mismo, la necesidad de una pedagogía orientada a la acción y transformadora, que potencie el aprendizaje autoregulado, la participación y la colaboración, la resolución de problemas utilizando un abordaje interdisciplinar y trans-disciplinar, y que ponga en juego distintos tipos de aprendizaje en contextos formales, no formales e informales. Porque, afirma, "sólo estos enfoques pedagógicos permiten la formación de las competencias clave necesarias para promover el desarrollo sostenible" (Unesco, 2017, p. 7).

A la hora de definir qué entiende por competencias, el texto las califica de atributos individuales específicos necesarios para la acción y la auto-organización en contextos y situaciones complejas. Incluyen elementos cognitivos, afectivos, volitivos y motivacionales. Y son, por tanto, resultado de la interacción de conocimientos, capacidades y habilidades, motivaciones y actitudes (disposiciones afectivas).

Las competencias se adquieren en la práctica, mediante la acción, sobre la base de la experiencia y la reflexión. Algunas de ellas, son competencias clave, necesarias para todos los estudiantes, de todas las edades y en todo el mundo (adquiridas en diferentes niveles, apropiados a la edad). Se caracterizan por ser transversales, multifuncionales e inespecíficas; es decir, no están vinculadas a contextos y situaciones predeterminadas. Unesco (2017, p. 10) las denomina "competencias clave de la sostenibilidad" para enfatizar que los ciudadanos las necesitan para actuar con éxito ante los complejos desafíos socioecológicos actuales. Son relevantes para todos los ODS y también permiten a los individuos relacionar cada uno de los ODS con los restantes y así ver la gran panorámica que está integrada por todos ellos.

A raíz de la Cumbre "Rio+20", Unesco señaló como tales competencias las cuatro siguientes: "análisis crítico, reflexión sistémica, toma de decisiones colaborativa, y sentido de la responsabilidad hacia las generaciones presentes y futuras" (Unesco, 2014, p. 12). Sin embargo, en la publicación posterior que nos está sirviendo de referente (Unesco, 2017) -ya en el marco de la Agenda 2030- el elenco se amplía hasta ocho (figura 1), incluyendo en el grupo cinco nuevas y evitando la última de las entonces señaladas, precisamente aquella que, desde nuestro punto de vista, refleja el más profundo sentido ético de la noción de sostenibilidad.

Efectivamente, la supresión de la mención explícita, entre las competencias clave, del sentido de responsabilidad inter e intra-generacional, aleja de los espacios de formación un principio y valor central de la sostenibilidad desde que el concepto de desarrollo sostenible fuera acuñado y difundido por el Informe Brundtland (WCED, 1987). Y también se descuida el llamamiento de la Carta de la Tierra, considerada por la propia Unesco (2003, p. 36) un referente de la educación para el desarrollo sostenible, cuyo texto señala sin ambages: todos compartimos una responsabilidad hacia el bienestar presente y futuro de la familia humana y del mundo viviente en su amplitud y nos insta a asegurar que los frutos y la belleza de la Tierra se preserven para las generaciones presentes y futuras (ECI, 2000). 


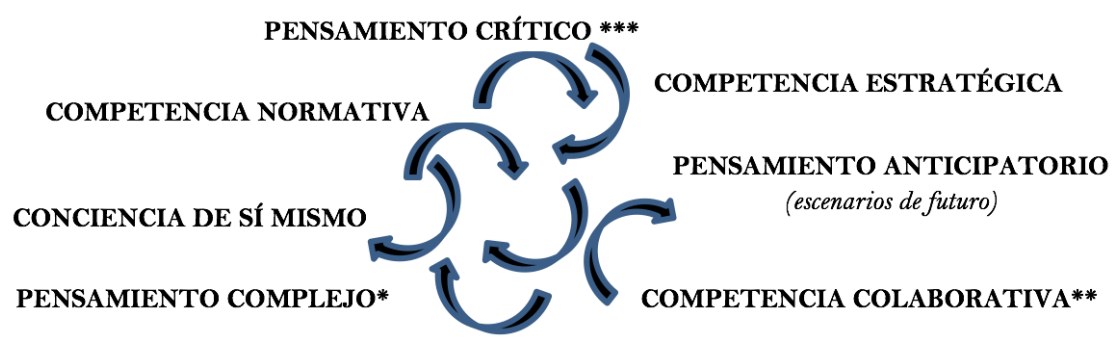

COMPETENCIA PARA LA RESOLUCIÓN INTEGRADA DE PROBLEMAS

Gráfico 1. Competencias para el logro de los ODS

Notas: * Equiparable a "reflexión sistémica" (Unesco, 2014, p.12); ** Equiparable a "toma de decisiones colaborativa” (Unesco, 2014, p.12); *** Equiparable a “análisis crítico” (Unesco, 2014, p.12)

Fuente: Elaboración propia basada en Unesco (2017, p. 10)

Esta laguna resulta inadmisible a la luz del enfoque de la justicia ambiental en cuyo marco el principio de responsabilidad ampliada (inter-generacional e inter-específica) es uno de los pilares básicos del entramado axiológico, sólidamente anclado en los derechos humanos universales, que da identidad al enfoque. Aquí se encuentra una debilidad de hondo calado que, desde la perspectiva de dicho enfoque, presentan las orientaciones de Unesco para el logro de los ODS.

Esta limitación no parece, sin embargo, insalvable. Bien al contrario, la re-inclusión de la competencia olvidada contribuiría a reforzar la base axiológica de la educación para el logro de los ODS, potenciando un proyecto humanizador de mayor consistencia. Hacer operativa esta re-inclusión tan solo exige que el diseño pedagógico de los procesos formativos se realice teniendo en cuenta no solo los valores de la ética ambiental sino abordando específicamente, con una perspectiva glocal y a largo plazo, el análisis crítico de las problemáticas referidas a cada uno de los ODS, sus causas estructurales y personales, y las estrategias para desactivarlas.

En definitiva, una educación para el desarrollo sostenible y la justicia ambiental, ineludiblemente ha de interpelar a los estudiantes y comprometerles, más allá de la búsqueda del eficientismo socio-ecológico, con la transformación integral de la sociedad. Porque, como acertadamente destaca Gadotti (Gentili, 2012), es preciso involucrar a la escuela en la comprensión de que toda agresión al medio ambiente es siempre una agresión social que tiene al planeta como destinatario, pero que impacta de forma mucho más brutal, por ejemplo, contra los más pobres.

En el enfoque de la justicia ambiental priman la ética y los valores morales sobre cualquier otra consideración. Cabe, por tanto, considerar que la justicia ambiental es un movimiento germen de una aún hoy incipiente ciudadanía para la transición ecológica y social hacia la sostenibilidad, cuya formación ha de ser abordada en todos los niveles del sistema educativo y, como veremos en el próximo apartado, no está exenta de dificultades. 


\section{El reto educativo capital: La formación de la ciudadanía}

En respuesta a la posición de la Asamblea General de las Naciones Unidas cuando declara: estamos decididos a tomar las medidas audaces y transformativas que se necesitan urgentemente para reconducir al mundo por el camino de la sostenibilidad y la resiliencia (ONU, 2015), Unesco está impulsando lo que denomina ciudadanía global, una ciudadanía explícitamente comprometida con el desarrollo sostenible (Unesco, 2015, 2016), posición que este alto organismo también mantiene al señalar los objetivos de aprendizaje para el logro de los ODS (Unesco, 2017).

Se trata de un concepto controvertido, en el cual la propia Unesco "reconoce matices y distintas perspectivas que se reflejan en diferentes adjetivaciones y términos; entre ellos, además de ciudadanía global, ciudadanía mundial, ciudadanía sin fronteras o ciudadanía planetaria" (Unesco, 2016, p. 15). Lo común en los numerosos textos que difunden la interpretación del concepto respaldada por Unesco, es que hacen alusión a una ciudadanía capaz de resolver activamente los desafíos mundiales y contribuir a un mundo más pacífico, tolerante, inclusivo y seguro; una ciudadanía que asume un rol activo, tanto local como globalmente.

Esta posición de Unesco supone un avance con relación al concepto de ciudadanía clásica. Además, como es habitual en la postura que adoptan los organismos internacionales, el concepto de ciudadanía global tiene la virtualidad de ofrecer una amplia horquilla para la interpretación, los matices ideológicos y las peculiaridades de los diferentes contextos. Sin embargo, no logra subsumir las controversias que generan las miradas alternativas derivadas de la específica preocupación que, desde los años sesenta del pasado siglo, viene emergiendo en paralelo con la intensificación de las problemáticas socio-ecológicas.

Efectivamente, el concepto de ciudadanía global muestra claras limitaciones al confrontarse con las propuestas de los movimientos ambientalistas y por la justicia ambiental, en cuyo seno se han acuñado toda una constelación de conceptos y denominaciones alternativas (Dobson, 2001, 2003, 2005). En un primer momento, se reivindicó la ciudadanía ambiental, entendida exclusivamente en términos de derechos ambientales realizados en la esfera pública y en el contexto del estado-nación -con hincapié en los derechos individuales- aunque, prácticamente de forma simultánea, surgieron conceptos como ciudadanía ecológica, o ciudadanía planetaria, ambos ya en el terreno de lo que se conoce como meta-ciudadanías. Estas últimas suponen un cambio de perspectiva, más allá de las posturas convencionales de la ciudadanía clásica, y, en consecuencia, implican un abordaje alternativo de los aspectos ambientales (Gudynas, 2009), por lo cual permiten atender más radicalmente a la formación de la ciudadanía en los principios y valores de la sostenibilidad.

Entre las características específicas comunes a los conceptos de ciudadanía que se integran en la innovadora categoría de las meta-ciudadanías cabe destacar tres principales. En primer lugar, acentúan el énfasis más en las obligaciones que en los derechos, haciéndolas extensivas a seres lejanos en el tiempo y el espacio (asincronía y aterritorialidad). Además, proclaman la responsabilidad diferenciada (asimétrica) de cada individuo en función de su capacidad causativa. Y, en tercer lugar, trascienden la distinción público-privado en el ejercicio de los deberes y derechos; tienen el bien común como prioridad. 
De entre las denominaciones que se han acuñado desde esta perspectiva, el término ciudadanía planetaria refleja con especial acierto el tipo de ciudadanía que la educación ha de formar para el logro de los ODS en el marco de la justicia ambiental y social. El adjetivo explícitamente alude al espacio físico de todo el planeta, rememorando la pertenencia del ser humano a una única comunidad de vida, y promoviendo, en este sentido, una consideración holística del ejercicio de la ciudadanía enraizada en el ecosistema (MurgaMenoyo y Novo, 2017).

Por otra parte, es un concepto que se ha forjado con sólidos mimbres que dan consistencia a su fundamentación teórica y axiológica. Tiene sus raíces en el ámbito de la Ecopedagogía (Boff, 2001, 2008; Gadotti, 2002, 2003; Gutiérrez Pérez, 2003; Gutiérrez Pérez y Prado, 2004), enfoque que asume, simultáneamente, los planteamientos de la Carta de la Tierra (ECI, 2000), el modelo pedagógico de Paulo Freire $(1967,1990)$ y las propuestas de Edgard Morín (1982, 2001).

En todo caso, sea cual fuere la denominación adoptada -ciudadanía global, ciudadanía verde, eco-ciudadanía, ciudadanía planetaria, ciudadanía ecológica; o cualquier otra designación- desde la perspectiva de la sostenibilidad el concepto de ciudadanía se concibe en términos de ciudadanía práctica, capaz de contribuir al buen vivir comunitario y de vincular las políticas sobre la naturaleza con cuestiones básicas de democracia y justicia. Porque, como acertadamente reclaman Latta y Wittman (2012,113), es preciso ir más allá de la limitada noción de ciudadanía verde o ambiental y su énfasis normativo para promover la sostenibilidad. Es necesaria una concepción de ciudadanía más profundamente política y más integrada desde el punto de vista ecológico. Una concepción que considere a los ciudadanos y la naturaleza elementos dinámicos, en confrontación y condicionados mutuamente dentro de la política del medio ambiente.

\section{Discusión y conclusiones}

A partir de lo hasta aquí expuesto, parece no solo posible sino conveniente -y hasta imprescindible- que las propuestas formativas orientadas al logro de la Agenda 2030 nunca descuiden los principios del movimiento por la justicia ambiental, en el núcleo más radical de la sostenibilidad. De igual modo, los proyectos educativos en el marco de dicho movimiento se verán fortalecidos si amplían su horizonte teniendo en cuenta los ODS, lo cual les permitiría una visión más holística y fundamentada de sus planteamientos y finalidad.

Meta central de estas propuestas ha de ser la formación para el ejercicio de las competencias clave en sostenibilidad, las cuales, por su propia naturaleza, presentan múltiples facetas interrelacionadas. Por este motivo, para la calidad de los procesos formativos, es preciso definir operativamente cada una de ellas, reconocer las capacidades que se requieren para su desempeño, identificar sus componentes (habilidades, conocimientos, valores, actitudes, destrezas, etc.). Y, finalmente, asociar a estos últimos los indicadores (conductas: verbales, motoras, escritas, etc.) cuya observación permite inferir la existencia de las correspondientes capacidades (Murga-Menoyo, 2015).

En el cuadro 1 se recogen las competencias clave en sostenibilidad que Unesco destaca (2017), así como las capacidades significativas que dicho organismo incluye en ellas e, ineludiblemente para que la propuesta pueda ser coherente con las finalidades de la justicia ambiental, se recupera la competencia enunciada previamente por dicho organismo 
(Unesco, 2014), sentido de la responsabilidad hacia las generaciones presentes y futuras (p. 12). Hoy la formación de la ciudadanía desde esta perspectiva se abre camino como objetivo universal de la educación; en todos sus niveles, ámbitos y modalidades.

Cuadro 1. Competencias para la sostenibilidad (en el marco de la Agenda 2030 y la Justicia ambiental)

\begin{tabular}{|c|c|}
\hline COMPETENCIAS CLAVE & CAPACIDADES \\
\hline $\begin{array}{l}\text { Pensamiento complejo } * * \\
\text { Reflexión sistémica } *\end{array}$ & $\begin{array}{l}\text { Capacidad de reconocer y entender las relaciones; } \\
\text { analizar sistemas complejos; pensar en cómo los sistemas } \\
\text { se insertan en diferentes dominios y escalas; y hacer } \\
\text { frente a la incertidumbre }\end{array}$ \\
\hline $\begin{array}{l}\text { Pensamiento anticipatorio } \\
\text { (escenarios futuros) } * *\end{array}$ & $\begin{array}{l}\text { Capacidad para comprender y evaluar múltiples } \\
\text { escenarios futuros - posible, probable y deseable; } \\
\text { concebir una visión de futuro propia y alternativa; aplicar } \\
\text { el principio de precaución; evaluar las consecuencias de } \\
\text { las acciones; y lidiar con los riesgos y los cambios }\end{array}$ \\
\hline Competencia normativa $* *$ & $\begin{array}{l}\text { Capacidad para entender las normas y valores que } \\
\text { subyacen a la propia conducta y consensuar valores, } \\
\text { principios, objetivos y metas de sostenibilidad, en un } \\
\text { contexto de conflicto de intereses, concesiones } \\
\text { recíprocas, conocimiento incierto y contradicción }\end{array}$ \\
\hline Competencia estratégica ** & $\begin{array}{l}\text { Capacidad para desarrollar y ejecutar colectivamente } \\
\text { acciones innovadoras que fomenten la sostenibilidad a } \\
\text { nivel local y más allá }\end{array}$ \\
\hline $\begin{array}{l}\text { Competencia colaborativa } * * \\
\text { Toma de decisiones colaborativa } *\end{array}$ & $\begin{array}{l}\text { Capacidad para aprender de los demás; comprender y } \\
\text { respetar las necesidades, perspectivas y acciones de otros } \\
\text { (empatía); comprender, relacionarse y ser sensibles a los } \\
\text { demás (liderazgo empático); hacer frente a los conflictos } \\
\text { en un grupo; y facilitar la colaboración y la resolución } \\
\text { participativa de problemas }\end{array}$ \\
\hline $\begin{array}{l}\text { Pensamiento crítico ** } \\
\text { Análisis crítico } *\end{array}$ & $\begin{array}{l}\text { Capacidad para cuestionar prácticas, opiniones y normas; } \\
\text { reflexionar sobre los propios valores, percepciones y } \\
\text { acciones; y tomar posición respecto al discurso de la } \\
\text { sostenibilidad }\end{array}$ \\
\hline Conciencia de sí mismo *** & $\begin{array}{l}\text { Capacidad para reflexionar sobre el propio papel en la } \\
\text { comunidad local y en la sociedad (global); valorar y } \\
\text { motivar continuamente las propias acciones y gestionar } \\
\text { los propios sentimientos y deseos }\end{array}$ \\
\hline $\begin{array}{l}\text { Competencia para la resolución } \\
\text { integrada de problemas } * *\end{array}$ & $\begin{array}{l}\text { Capacidad global de aplicar diferentes marcos para la } \\
\text { resolución de problemas complejos de sostenibilidad, } \\
\text { desarrollando soluciones viables, inclusivas y } \\
\text { equitativas, que promuevan el desarrollo sostenible y la } \\
\text { integración de todas las competencias mencionadas }\end{array}$ \\
\hline $\begin{array}{l}\text { Sentido de la responsabilidad con } \\
\text { las generaciones presentes y futuras } \\
\text { * }\end{array}$ & $\begin{array}{l}\text { Capacidad para comprender que toda agresión al medio } \\
\text { ambiente es siempre una agresión eco-social, de impacto } \\
\text { en el planeta, en la Humanidad e, incluso, en la especie } \\
\text { humana. Capacidad para un compromiso activo por la } \\
\text { justicia social y ambiental }\end{array}$ \\
\hline
\end{tabular}

Notas: * Unesco (2014). ** Unesco (2017).

Fuente: Elaboración propia.

Por otra parte, el movimiento por la justicia ambiental incluye entre sus finalidades la formación de activistas sociales, formación que exige no sólo la capacidad crítica sino el cultivo explícito de una actitud de indignación y rebeldía ante la falta de equidad, la indefensión de los excluidos y, en definitiva, la injusticia, en todas sus manifestaciones, así como hábitos de movilización para impedirla. Y, desde esta perspectiva, se enfatiza la educación como un acto político, orientado al empoderamiento de los más vulnerables y 
encaminado a la transformación social. Esta posición implica que los tres principales dominios que Unesco (2017) establece para categorizar los objetivos de aprendizaje cognitivo, socio-emocional y conductual- (p. 11) han de verse imbuidos en profundidad por una ética de la compasión y los cuidados, convertida en eje transversal del proceso formativo.

Una ética de estas características subyace en la Carta de la Tierra (ECI, 2000), código de buen hacer que, como anteriormente hemos comentado, la propia Unesco (2003, 36) avala como referente de la educación para el desarrollo sostenible. De su entramado axiológico, que religa los valores en una tupida malla, se deduce la necesidad de una educación inspirada en el pathos (Boff, 2012); es decir, en la sensibilidad humanitaria y la inteligencia emocional. La equidad, la austeridad, la compasión, la responsabilidad, la libertad, la solidaridad sincrónica (inter e intra-generacional, e inter-específica) y la solidaridad diacrónica, la espiritualidad, la co-responsabilidad en la promoción del bien común, la justicia social y económica, el diálogo, la diversidad biológica y cultural, la tolerancia, el valor de la vida independientemente de su utilidad, aparecen implícita o explícitamente en su texto (Murga-Menoyo, 2005, 2009). Todos ellos se convierten, desde esta perspectiva, en valores centrales de los procesos formativos.

Esta posición se ve reforzada al adoptar como referente una teoría de las necesidades basada en la justicia (con el planeta y con los pobres de la Tierra), que establezca una clara distinción entre necesidades y deseos a la vez que reconoce el potencial de los satisfactores como mediadores entre las necesidades (interioridad) y los bienes (exterioridad) (MaxNeef y Smith, 2014). En torno a las necesidades y sus satisfactores se abre un amplio espacio de libertad y autonomía personal en el cual la educación tiene una extensa cancha de actuación ante los significativos efectos que la elección de un satisfactor entre varios posibles podría llegar a tener sobre la propia vida y la ajena, y sobre el ecosistema local y global. Los satisfactores tienen efectos sistémicos sobre la realidad vital y social de las personas y las comunidades.

Esta relación dialéctica entre necesidades, satisfactores y bienes se manifiesta en los diferentes estilos de vida, cuyo análisis permite evaluar el buen vivir de una sociedad, así como su viabilidad futura. Y para ello, como acertadamente señala Elizalde (2003),

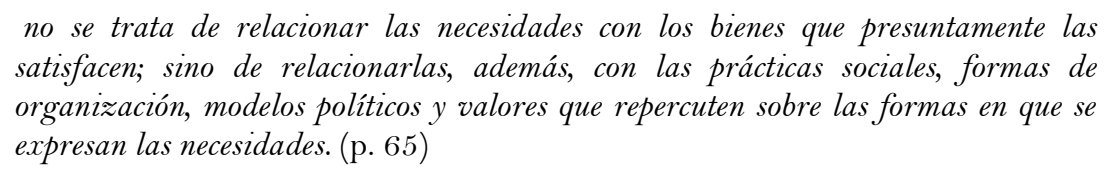

En consecuencia, los procesos formativos para el logro de la Agenda 2030 y la justicia ambiental, ineludiblemente, han de promover el rechazo de los pseudo-satisfactores (satisfactores destructores, o inhibidores); y potenciar, paralelamente, los sinérgicos, es decir, aquellos que contribuyen a que la satisfacción de las necesidades sea el motor del desarrollo y la evolución humana, nunca su meta. La formación de las competencias clave para la sostenibilidad recogidas en la tabla 1 facilita, sin duda, este planteamiento.

Respecto a los contenidos que Unesco (2017) pide incluir en el currículo para el logro de los ODS, ninguno de ellos supone un inconveniente por incompatibilidad con las finalidades de la justicia ambiental; simplemente, algunos no se encuentran aún en el punto de mira del movimiento. Sin embargo, su consideración ampliaría el abanico de las problemáticas tradicionales que este último viene abordando, e impulsaría en su seno un enfoque amplio del concepto de medio ambiente, en coherencia con los principios y valores 
de la sostenibilidad. Así lo destacan nítidamente las Naciones Unidas al afirmar que los ODS "son de carácter integrado e indivisible y conjugan las tres dimensiones del desarrollo sostenible: económica, social y ambiental” (ONU, 2015, p. 2).

Este planteamiento exige a la educación un enfoque metodológico que permita abordar las cuestiones desde perspectivas múltiples, contando para ello con el concurso de las diferentes disciplinas. La formación de competencias clave para la sostenibilidad tiene un significativo foco de atención en la trama que articula las problemáticas socio-ecológicas y humanas, siempre de naturaleza multidimensional. Comprender las interconexiones, complementariedades y perspectivas poliédricas es obligado para alcanzar una visión integrada de la realidad.

Pero, junto a los procedimientos interdisciplinares y sistémicos, se requieren metodologías socio-afectivas, adecuadas para afianzar los valores y las actitudes que reiteradamente venimos defendiendo como componentes ineludibles de la educación para el desarrollo sostenible (Murga-Menoyo y Novo, 2014). Como bien señala la Unesco (2017), es preciso impulsar una pedagogía abocada a la acción y la resolución de problemas, con un abordaje interdisciplinar y trans-disciplinar, que prime la dimensión aplicada y potencie el aprendizaje autorregulado en todo tipo de contextos, formales, no formales e informales. Estas características son adecuadas no solo para avanzar en las metas de la Agenda 2030 sino también para el tipo de la ciudadanía que demanda la justicia ambiental.

En definitiva, las afinidades que se han puesto de manifiesto permiten afirmar que las posibles diferencias entre el enfoque de la Agenda 2030 y el movimiento por la justicia ambiental son, en la mayoría de los casos, más cuestión de grado que radicales. Afirmación que no niega la significativa prioridad de la preocupación por la justicia social como elemento diferenciador específico del enfoque de la justicia ambiental; prioridad muy tibiamente considerada por una amplia mayoría de los que se auto-sitúan en el enfoque de la sostenibilidad. Sin embargo, el marco conceptual de ambos enfoques permite, sin duda, abordar un tipo de formación que desafíe la creciente mercantilización de la educación y, como defiende Gadotti (2012, p. 21), considere prioritario "educar para cambiar radicalmente nuestra manera de producir y de reproducir nuestra existencia en el planeta”.

\section{Referencias}

Arriaga Legarda, A. y Pardo Buendía, M. P. (2011). Justicia ambiental. El estado de la cuestión. Revista Internacional de Sociología, 69(3), 627-648.

Banco Mundial. (2017). Atlas of sustainable development goals 2017: From world development indicators. Washington, DC: World Bank.

Boff, L. (2001). Ética planetaria desde el gran sur. Madrid: Editorial Trotta.

Boff, L. (2008). La opción-tierra. Santander: Sal Térrea.

Boff, L. (2012). El cuidado necesario. Madrid: Trotta

Caride Gómez, J. A. (2009). Nuevas perspectivas para un futuro viable: Los objetivos de desarrollo del milenio. Revista de Educación, núm. extraordinario, 77-98.

Díaz-Salazar, R. (2016). Educación y cambio ecosocial. Del yo interior al activismo ciudadano. Madrid: PPC. 
Dobson, A. (1998). Justice and the environment: Conceptions of environmental sustainability and theories of distributive justice. Londres: Oxford Scholarship Online.

Dobson, A. (2001). Ciudadanía ecológica: ¿Una influencia desestabilizadora? Isegoría, 24, 167-187.

Dobson, A. (2003). Citizenship and the environment. Oxford: Oxford University Press.

Dobson, A. (2005). Ciudadanía ecológica. Isegoría, 32, 47-62.

ECI. (2000). La carta de la Tierra. Costa Rica: ECI.

Elizalde, A. (2003). Desarrollo humano y ética para la sustentabilidad. Santiago de Chile: Universidad Bolivariana.

Espinosa, A (2012). La justicia ambiental, hacia la igualdad en el disfrute del derecho a un medio ambiente sano. Universitas. Revista de Filosofía, Derecho y Política, 16, 51-77.

Freire, P. (1967). La educación como práctica de la libertad. Río de Janeiro: Paz e Terra.

Freire, P. (1990). La naturaleza política de la educación. Barcelona: Paidós.

Gadotti, M. (2002). Pedagogía de la Tierra. Buenos Aires: Siglo XXI.

Gadotti, M. (2003). Perspectivas actuales de la educación. Ciudad de México: Siglo XXI Editores.

Gadotti, M. (2012). Justicia ambiental y educación. América Latina en Movimiento, 472, 18-21.

Gentili, P. (20 de enero de 2012). Justicia social, justicia ambiental y educación. Un diálogo con Moacir Gadotti sobre el Foro Social de Porto Alegre. El País. Recuperado de https://elpais.com/elpais/2012/01/30/contrapuntos/1327887533_132788.html

Gil Pérez, D. y Vilches, A. (2017). Educación para la sostenibilidad y educación en Derechos Humanos: dos campos que deben vincularse. Teoría de la Educación. Revista Interuniversitaria, 29(1), 79-100.

Gudynas, E. (2009). Ciudadanía ambiental y meta-ciudadanías ecológicas: Revisión y alternativas en América Latina. Desenvolvimento e Meio Ambiente, 19, 53-72.

Gutiérrez Pérez, F. (2003). Ciudadanía planetaria. En J. Martínez (Ed.), Ciudadanía, poder y educación (pp. 34-65). Barcelona: Grao.

Gutiérrez Pérez, F. y Prado, C. (2004). Ecopedagogía y ciudadanía planetaria. Valencia: Editorial Diálogos.

Helliwell, J., Layard, R. y Sachs, J. (2016). World happiness report 2016. Nueva York, NY: Sustainable Development Solutions Network.

IPPC. (2014). Climate change 2014. Synthesis report. Ginebra: IPCC.

Latta, A. y Wittman, H. (2012). Environment and citizenship in Latin America: A new paradigm for theory and practice. European Review of Latin American and Caribbean Studies, 89, 107116.

Martínez Alier, J. (2004). El ecologismo de los pobres. Conflictos ambientales y lenguajes de valoración. Barcelona: Icaria.

Max-Neef, M. y Smith, P. B. (2014). La economía desenmascarada. Del poder y la codicia a la compasión y el bien común. Barcelona: Icaria.

Millennium Ecosystem Assessment. (2005). Ecosystems and human well-being: Synthesis. Washington, DC: Island Press.

Morín, E. (1982). Science avec conscience. París: Fayard.

Morín, E. (2001). Los siete saberes necesarios para la educación del futuro. Barcelona: Paidós. 
Moreno Jiménez, M. A. (2007). ¿Está equitativamente repartida la contaminación sonora urbana? Una evaluación desde el principio de justicia ambiental en la ciudad de Madrid. Estudios Geográficos, 68(263), 595-626.

Moreno Jiménez, M. A. (2010). Justicia ambiental. Del concepto a la aplicación en análisis de políticas y planificación territoriales. Scripta Nova. Revista Electrónica de Geografía y Ciencias Sociales, 14(316), art 3.

Moreno Jiménez, M. A. y Cañada Torrecilla, R. (2007). Justicia ambiental y contaminación atmosférica por dióxido de azufre en Madrid: Análisis espacio-temporal y valoración con sistemas de información geográfica. Boletín de la Asociación de Geógrafos Españoles, 44, 301324.

Murga-Menoyo, M. A. (2005). La carta de la Tierra. Conceptos, principios y valores para la educación. En M. A. Murga-Menoyo y P. Quicios García, La educación en el siglo XXI. Nuevos horizontes (pp. 55-92). Madrid: Dykinson.

Murga-Menoyo, M. A. (2009). La carta de la Tierra: Un referente de la década por la educación para el desarrollo sostenible. Revista de Educación, num. extraordinario, 239-262.

Murga-Menoyo, M. A. (2015). Competencias para el desarrollo sostenible: Las capacidades, actitudes y valores meta de la educación en el marco de la agenda global post-2015. Foro de Educación, 13(19), 55-83.

Murga-Menoyo, M. A. y Novo, M. (2014). Sostenibilizar el curriculum. La carta de la Tierra como marco teórico. Edetania, 46, 163-179.

Murga-Menoyo, M. A. y Novo, M. (2017). Sostenibilidad, desarrollo “glocal” y ciudadanía planetaria. Referentes de una pedagogía para el desarrollo sostenible. Teoría de la Educación. Revista Interuniversitaria, 29(1), 55-78.

ONU. (1972). Report of the united nations conference on the human environment. Recuperado de http://www.un-documents.net/aconf48-14r1.pdf

ONU. (2002). Report of the world summit on sustainable development. Recuperado de http://www.undocuments.net/aconf199-20.pdf

ONU. (2012). Report of the united nations conference on sustainable development. Recuperado de https:// sustainabledevelopment.un.org/index.php?page=view\&type $=111 \& \mathrm{nr}=1358 \& \mathrm{men}$ $\mathrm{u}=35$

ONU. (2015). Transformar nuestro mundo: La agenda 2030 para el desarrollo sostenible. Recuperado de http://unctad.org/meetings/es/SessionalDocuments/ares7od1_es.pdf

Sachs, J., Schmidt-Traub, G., Kroll, C., Durand-Delacre, D. y Teksoz, K. (2017). SDG Index and Dashboards Report 2017. Nueva York, NY: SDSN.

UNEP. (2011). Keeping track of our changing environment: From Rio to Rio+20 (1992-2012). Nairobi: UNEP.

Unesco. (2003). Actas de la 32 conferencias general. París: Unesco.

Unesco. (2014). Roadmap for implementing the global action programme on education for sustainable development. Recuperado de https: / / sustainabledevelopment.un.org/index.php?page $=$ view $\&$ type $=400 \& \mathrm{nr}=1674 \& \mathrm{men}$ $\mathrm{u}=35$

Unesco. (2015). Educación para la ciudadanía mundial. Temas y objetivos de aprendizaje. Recuperado de http://www.unesco.org/new/es/education/resources/in-focus-articles/globalcitizenship-education/ 
Unesco. (2016). Global citizenship education, preparing learners for the challenges of the 21st century. Recuperado de https://es.unesco.org/node/ 185462

Unesco. (2017). Education for sustainable development goals. Learning objectives. Recuperado de http://www.unesco.org/new/en/brasilia/about-this-office/single-

view/news/education_for_sustainable_development_goals_learning_object/

Vicente Jiménez, T. (2002). La exigencia de un modelo de justicia para la humanidad y el planeta, Anales de Derecho, 20, 155-162.

Wenz, P. S. (1988). Environmental justice. Nueva York, NY: State University of New York Press.

WCED-World Commission on Environment and Development. (1987). Our common future. Recuperado de http://www.un-documents.net/our-common-future.pdf

\section{Breve $\mathrm{CV}$ de la autora}

\section{$M^{a}$ Ángeles Murga-Menoyo}

Profesora de la Facultad de Educación y de la Escuela Internacional de Doctorado de la UNED, desde 1996 es miembro de la Cátedra Unesco de Educación Ambiental y Desarrollo Sostenible. Participante habitual en congresos y foros científicos nacionales e internacionales, cuenta con más de sesenta publicaciones: libros, capítulos de libro y artículos científicos. Es miembro del Comité Científico de la Revista Bordón (Sociedad Española de Pedagogía) y colabora asiduamente como evaluadora externa en revistas científicas. Actualmente lidera un proyecto de investigación interuniversitario financiado por el Ministerio de Economía, Industria y Competitividad del Gobierno de España, orientado a la sostenibilización curricular de la formación del profesorado. Ha desempeñado el cargo de Directora del Departamento de Teoría de la Educación y Pedagogía Social (2003-2015) y representante de la UNED en CRUE-Sostenibilidad (2012-2016). ORCID ID: 0000-0001-8779-6192. Email: mmurga@edu.uned.es 